\title{
Three Types of Episodic Associations for the Semantic/Syntactic/Episodic Model of Language Prospective in Applications to the Statistical Translation
}

\author{
Zi-Jian Cai \\ CaiFortune Consulting, Suzhou, China \\ Email: hrsh8@126.com
}

How to cite this paper: Cai, Z.-J. (2017) Three Types of Episodic Associations for the Semantic/Syntactic/Episodic Model of Language Prospective in Applications to the Statistical Translation. Open Access Library Journal, 4: e3830.

https://doi.org/10.4236/oalib.1103830

Received: July 16, 2017

Accepted: August 4, 2017

Published: August 7, 2017

Copyright (๑) 2017 by author and Open Access Library Inc.

This work is licensed under the Creative Commons Attribution International License (CC BY 4.0).

http://creativecommons.org/licenses/by/4.0/ (c) (i) Open Access

\begin{abstract}
Recently, it was proposed by Cai a new semantic/syntactic/episodic model of language encompassing the sentential meanings, while deriving three corresponding principles from it for machine translation, respectively as first to establish the dictionary of words/phrases, second to translate the grammar, and third to determine the meanings of some words/phrases of multiple meanings by statistical translation. In this article, it is discovered three types of episodic associations for this linguistic model, prospective in applications to statistical translation, as: 1) It is classified the living/natural words and phrases of multiple meanings by behavior, adopting both the zoological/organizational/physical/categorical and affective/behavioral/logic/characteristic/changing characters to classify the nouns and verbs, the affective/behavioral/logic/characteristic/changing/spatial/temporal characters to the adjectives and adverbs, helpful to discern the meanings of them using these episodic associations with others within the sentence. 2) Likewise, it is classified the sentence/paragraph into the category of natural/social subjects like physics, biology, art, economy, etc., which was improved by the Chinese people in television from my original sentential/thematic category. 3) It is suggested to collect the frequent wordpairs during statistical translation, such as "bank money", "war declaration", etc., helpful to determine the episodic associations of some prepositions or terminal "which" clauses. It is suggested to use word episodic symbolization to apply them to computer. It is therefore improved the third principle of machine translation as third to determine the meanings of some words/ phrases of multiple meanings by episodic associations with others using the behavioral classification of words, the categorization of sentence/paragraph and the collection of frequent word-pairs.
\end{abstract}




\section{Subject Areas}

Artificial Intelligence, Information and Communication Theory and Algorithms, Linguistics, Neuroscience

\section{Keywords}

Semantic/Syntactic/Episodic Linguistic Model, Behavioral Classification of Words, Sentential/Paragraphic Categorization, Frequent Word-Pairs, Word Episodic Symbolization, Statistical Translation

\section{Introduction}

The natural language of humans is organized from word to sentence to story, making the language able to contain and deliver almost all kinds of information and intention at ease [1].

Recently, the author proposed a new semantic/syntactic/episodic model of three kinds of linguistic processes in brain, so as to encompass the sentential meanings to the linguistic processes [1] [2].

From this semantic/syntactic/episodic linguistic model, the author has also derived three principles for guidance of software design of translation machines [2], as followings: 1) Principle 1 is the necessity to establish the dictionary for translation of words and phrases in machine translation. 2) Principle 2 is the necessity to install the linguistic grammar for reading and forming the sentence from words and phrases in machine translation, arranging such parts of speech as noun, verb and adjective into order. 3) Principle 3 is the necessity to check with statistics for the words and phrases of multiple meanings and figure out one correct meaning of them in machine translation.

The Principles 1 - 2 of machine translation are regular and fixed in contents or rules, while the Principle 3 nowadays popularly present as the statistical translation requires to store and rectify the statistical concurrence of word pairs with the accumulation of translation. It is relatively most flexible in application, and accordingly it would be helpful if provided with some common methods to help coordinate the technological applications. In this article, it is newly demonstrated three types of episodic associations for the semantic/syntactic/episodic linguistic model to prospectively help and improve the statistical translation nowadays, while other improvements to statistical translation from visual, auditory inputs and so on are not considered in this paper.

\section{The Brief Semantic/Syntactic/Episodic Model of Language}

Recently, Cai extended the present declarative/procedural model of Ullman and Pinker [1] [3] [4] [5] underlying two kinds of linguistic processes to a new semantic/syntactic/episodic model of three kinds of linguistic processes in brain [1] [2], able to encompass the sentential meanings to the linguistic processes that 
the declarative/procedural model of Ullman and Pinker has neglected [1] [2] [3] [4] [5].

Pinker, Ullman and so on integrated a declarative/procedural model for linguistic processing in brain. They suggested that the linguistic lexicon of wordspecific knowledge should subserve the storage of meanings of words and phrases, and depend on the temporal-lobe substrates of declarative memory for the storage and usage of facts and events [1] [3] [4] [5], while the linguistic grammar should subserve the sequential combination of lexical items by procedural rules into complex sentences, and depend on a network of specific frontal, parietal, basal-ganglia and cerebellar structures of procedural memory in the brain [1] [3] [4] [5]. There are many evidences in support of this declarative/ procedural model of language [1] [3] [4] [5].

To extend this declarative/procedural model to a new semantic/syntactic/episodic model of three linguistic processes in brain, Cai added that, besides the declarative/procedural processes of language, the episodic coordination of cortical modality by reticular formations was also required for language performance and organization in addition [1] [2]. Many neurobiological evidences supported this suggestion [1] [2], as in the followings: 1) The reticular dopaminergic system may be involved in the linguistic modality organization, as the dopaminergic antagonists [1] [2] [6] [7] alleviate while the dopaminergic genes [1] [2] [8] phenotype the stuttering. 2) From the drugs in many assisting therapies of aphasia, it is implicated that the ascending reticular cholinergic projections, noradrenergic projections and nonspecific activating projections from intralaminar thalamic nuclei may participate in episodic coordination of cortical modalities in linguistic processes [1] [2] [9] [10]. 3) It is evidenced by numerous reports that the gamma bands of high frequency, subject to modulation by the reticular cholinergic system [11] [12], associate with the word congruency at sentential level [1] [2] [13] [14] [15], and the episodic coordination of cortical modalities.

Now that it is well evidenced for the episodic coordination of cortical modalities at the sentential level, it is reasonable to in further consider it for story narration. Complex stories certainly require more episodic coordination of cortical modalities. Language disorders occur in narrative discourse skills in patients with various pathologies [16] [17] [18], demonstrating diversity in cortical modalities. Besides, stuttering is also a disorder of discourse sometimes, as narrative therapy is auxiliary to treatment of stuttering [19] [20]. These facts implicate that episodic modality coordination of cortices is also required at high level during story narration.

In summary, herein it is briefly demonstrated that there are three semantic/ syntactic/episodic linguistic processes present in the human brain.

\section{Three Principles for Machine Translation Derived from the Semantic/Syntactic/Episodic Neurolinguistics}

At present, there are two types of machine translation, adopting two different 
strategies of design respectively. One is early in method following which to translate the individual words and grammar of sentence [21]. The difficulty of this type of machine translation lies in that many words have multiple meanings, so that the translation often makes mistakes due to this reason. Another is the recent method called as statistical machine translation [22] [23], in which the machine translation is made according to the statistical concurrence of words and contents. The difficulty of this type of machine translation lies in that the statistical concurrence of words and contents is various and limitless from collection, so that it is necessary to further find out some rules to make it more effective, which is difficult.

Because of the lack of scientific guidance from linguistics, the present machine translation has mostly chosen one of the two types of methods, and therefore only adopted two linguistic processes rather than the recently revealed three brain linguistic processes. In this regard, the results of machine translation are quite unsatisfactory at present, and the translational mistakes occur everywhere [21] [22] [23].

With the recent semantic/syntactic/episodic model of three linguistic processes [1], it is possible to derive three corresponding principles from them for guidance of software design of translation machines [2].

From word comprehension as remote declarative memory associations in brain, it can be formulated the first principle for design of translation machines as followings (Table 1):

Principle 1: Corresponding to the word comprehension as memory associations, it is necessary to establish the dictionary for translation of words and phrases in translation machine.

From grammatical rule as procedural memory in brain, it can be formulated the second principle for design of translation machines as followings:

Principle 2: Corresponding to the grammatical rule as procedural memory, it is necessary to install the linguistic grammar for reading and forming sentence from words and phrases in translation machine, arranging such parts of speech as noun, verb and adjective into order.

From episodic coordination of language in brain, it can be formulated the third principle for design of translation machines as followings:

Principle 3: Corresponding to the episodic coordination of language, it is necessary to check with statistics of concurrence for the words and phrases of multiple meanings, and figure out one correct meaning of them in translation machine.

Table 1. Three principles of machine translation from semantic/syntactic/episodic neurolinguistics.

\begin{tabular}{lcc}
\hline & Neurolinguistic processes in brain & Principles of machine translation \\
\hline Principle 1 & Semantics as memory associations & Make dictionary of words/phrases \\
Principle 2 & Syntax as procedural memory & Install linguistic grammar \\
Principle 3 & Episodic coordination of language & Statistically check word meanings \\
\hline
\end{tabular}




\section{Behavioral Classification of Living/Natural Words}

In this article, it is mainly considered the episodic association of words for the semantic/syntactic/episodic linguistic processes to prospectively improve the statistical translation.

To determine the meaning of a word or phrase of multiple meanings in the sentence or clause, the episodic association of the word with other words in the sentence or clause can provide some important clues. For example, for the word "charge" as noun, it can be interpreted as 1) the duty, 2) the necessity of payment, 3) the amount of money necessary to pay, or 4) the electrical character and quantity, quite different in meanings. Its meaning varies in episodic association with other words in the sentence or clause. Statistical translation with episodic association is helpful to determine one correct meaning.

It is useful to find some common clues for identification of the word or phrase of multiple meanings in the sentence or clause. The word "he" and "she" can think or behavior as a living life, whereas the word "it" cannot in most situations except it means an animal. This is the further classification of pronouns in the present English. Besides, the present verbs have also been further classified into $\mathrm{Vt}, \mathrm{Vi}$ and link Verb.

The author suggests that many words can similarly be further classified according to the characteristics of them to possibly behave as a living entity or a natural matter, just as the present pronouns "he", "she" and "it". With such further classification of words by behavior, it is helpful to determine the meaning of some words or phrases of multiple meanings in the sentence or clause.

With the above noun "charge" as example, its meanings as duty and the necessity of payment contain the zoological or organizational mind and behavior, while its meanings as the amount of payment and the electrical character or quantity do not contain the living meaning, but the natural character. Herein, it is demonstrated that this clue is useful to identify the meaning of noun "charge", even though not completely.

It is especially important to point out that all contents expressed in language can fall into a special category of sciences. At the individual level, all sentential contents can fall into either physical sciences or life sciences, while at the organizational level; all sentential contents can fall into either natural sciences or social sciences. In this regard, the physical and zoological category can contain all nouns at the individual level, while the categorical and organizational category can contain all nouns at the organizational level. For example, the noun "worker" is zoological, and the noun "team" is organizational, while the noun "hydrogen" is physical, and the noun "element" is categorical.

The nouns in the physical and zoological category adopt different verbs to manifest their behaviors respectively. The physical verbs can correspondingly be classified as characteristic, changing and consequential characters, while the zoological verbs can be classified as affective, behavioral and logic. Due to the logic nature of consequential character, herein it is classified the consequential 
character into logic too. For example, the noun "sun" is physical, the relevant verb "shine" is characteristic, the relevant verb "rotate" is changing, and the relevant verb "bring about" is logic. The noun "patient" is zoological, the relevant verb "fear" is affective, the relevant verb "cry" is behavioral, and the relevant verb "result from" is logic.

Because some nouns, such as "application", are derived from verbs, it is also appropriate to further classify the nouns using the verb classification above as affective/behavioral/logic/characteristic/changing. Therefore, all nouns can be classified into both zoological/organizational/physical/categorical and affective/ behavioral/logic/characteristic/changing characters. Likewise, the verbs can also resemble the nouns to be categorized into the zoological/organizational/physi$\mathrm{cal} /$ categorical characters, with the affective/behavioral/logic verbs belonging to zoological/organizational, and the characteristic/changing/logic verbs belonging to physical/categorical.

Now that it is adopted the behavioral classification of the living/natural nouns and verbs into both the zoological/organizational/physical/categorical and affective/behavioral/logic/characteristic/changing characteristics, it is also possible to classify the adjectives and adverbs on their behaviors. With the adverb "along" as example, it can be interpreted as 1) altogether, 2) following others, 3) forward, 4) future, which can be classified into the 1) characteristic, 2) behavioral, 3) spatial, 4) temporal characteristics respectively. Besides, such words as "horrible", "exciting" represent the affective psychology, such words as "aggressive", "diligent" represent the behavioral psychology, and such words as "consequent", "thus" represent the logic of the sentential meaning, which should be discerned apart. In these regards, the adjectives and adverbs can also be classified into the affective/behavioral/logic/characteristic/changing/spatial/temporal characters. Correspondingly, the characteristic/changing/logic/spatial/temporal characters are physical or natural, while the affective/behavioral/logic characters are zoological or social.

It is noted that the name of a person or pet is zoological in psychology, but is physical in body. Thus, the name of a person or pet can be either zoological or physical, while its meaning should be determined in association with the verb, adjective and adverb in the sentence as zoological or physical.

Herein, as shown in Table 2, it is classified the living/natural nouns and verbs into both the zoological/organizational/physical/categorical and affective/behavioral/logic/ characteristic/changing characteristics, the adjectives and adverbs into the affective/behavioral/logic/characteristic/changing/spatial/temporal characteristics. The advantages of the classifications are: (1) They result from the universal nature of words falling into either natural sciences or social sciences. (2) The behavioral classifications of words are applicable to all nouns, verbs, adjectives and adverbs, with the characteristic/changing/logic words associating with physical/ categorical, and the affective/behavioral/logic words associating with zoological/ organizational. 
Table 2. Behavioral classification of the living/natural words.

\begin{tabular}{ccc}
\hline & Physical/categorical words & Zoological/organizational words \\
\hline Noun & Characteristic/changing/logic & Affective/behavioral/logic \\
Verb & Characteristic/changing/logic & Affective/behavioral/logic \\
Adjective & Spatial/temporal/characteristic/changing/logic & Affective/behavioral/logic \\
Adverb & Spatial/temporal/characteristic/changing/logic & Affective/behavioral/logic \\
\hline
\end{tabular}

\section{Sentential/Paragraphic Categorization}

The words within one sentence mostly adapt to the meanings fitting the category of subject of the sentence, such as the physics, biology, art, economy, and so on, in the natural sciences or social sciences. Categorization of the sentential subject can certainly be adopted to help statistical translation. After the natural/social subject of the sentence is categorized as the physics, biology, art, economy, and so on, some words of multiple meanings can be determined to one correct meaning fitting the category of this subject falling in either the natural science or social science, therefore helpful to the statistical translation.

Still with the above noun "charge" as example, if the subject of sentence is categorized by other words as physics, chemistry, computer, and so on, then the meaning of "charge" is most likely to be the electrical character or quantity. Herein again, with this linguistic example it is demonstrated that the sentential categorization is useful to determine the meaning of "charge".

As mentioned above, complex story narration certainly belongs to the episodic linguistic process and requires more complex episodic coordination. Originally, I adopted the thematic subject of the whole essay or article to help determine the word meanings fitting the subject. However, the Chinese people in television, especially the old people's representatives in Beijing in television on January 11, 2017, strongly argued that they often deviated the main topic while making speech, so that the thematic subject should be improved to the paragraphic subject for the same purpose.

The paragraphic subjects are usually more complex than the sentential subject. Besides the natural/social subjects such as the physics, biology, art, economy, and so on, it is possible to extend the subject to the story of life, personal experience, environmental depiction, and so on successional situations.

\section{Collection of Frequent Word-Pairs}

In some situations, the episodic association of words or clauses in the sentence is very intricate. For instance, the prepositions like by, with, through and via can associate in meaning with either noun or verb in the sentence. It is necessary to refer to the frequent word-pairs to determine the episodic associations.

For example it is herein to compare two sentences as: 1) They agreed the war by declaration. 2) They agreed the war with consensus. Using the word-pair "war declaration", it is determined "by declaration" in sentence 1) to modify the 
noun "war", while using the word pair "consensus agree", it is determined "with consensus" in sentence 2) to modify the verb "agreed". Obviously, this example demonstrates that the collection of frequent word-pairs is necessary to the statistical translation of the episodic association of some prepositions.

For another instance, the terminal "which" clause may modify the last noun, and may also manifest the consequence of the former sentence. It is also necessary to use the frequent word-pairs to determine the episodic associations.

For example it is herein also to compare two sentences as: 1) Tomorrow I am going to the park, which is beautiful. 2) Tomorrow I am going to the park, which is expected. Using the word-pair "beautiful park", it is determined the "which" clause in sentence 1) to modify the noun "park", while using the word pair "I expected", it is determined the "which" clause in sentence 2) to modify the former sentence "Tomorrow I am going to the park". Obviously, this example demonstrates that the collection of frequent word-pairs is also necessary to the statistical translation of episodic associations of terminal "which" clauses.

\section{Word Episodic Symbolization for Computer Applications}

Each meaning of a word or phrase belongs the semantic meanings of the word. For the words and phrases of multiple meanings, there are several semantic meanings of the words/phrases, with each of them belonging to their own behavioral classification and subject category as well as possessing their own wordpairs with others, manifesting three different types of episodic associations.

It is necessary to consider how to implement the application of them to computer. These episodic associations may be easily processed in computer as various databanks for SQL or Oracle. For the behavioral classification and subject category of words, the databank of semantic translation can be designed to contain and promote various relevant episodic associations, as illustrated in the following paragraph. For the collection of frequent word-pairs, they can be collected and stored directly in the specific databank of frequent word-pairs, separate from the data bank for behavioral classification and subject category.

The databank for storing and processing the behavioral classification and subject category of words deserves special demonstration. For a word or phrase of multiple meanings, herein it is recommended to use the symbol $\perp$ to separate the multiple meanings. For example, the noun "charge" has several meanings as "duty $\perp$ money-payment $\perp$ payment-amount $\perp$ electrical-quantity". With this symbol, a word of multiple meanings can be processed as if one data including all meanings in the databank.

Herein it is recommended to use another symbol $<>$ to characterize the behavioral classification and subject category of each meaning of the word or phrase of multiple meanings. For example, the noun "charge" can be better stored as one complex data in databank as "duty <zoological $><$ organizational $><$ behavioral $>\perp$ money-payment $<$ zoological $><$ organizational $><$ behavioral $>\perp$ payment-amount $<$ physical $><$ categorical $><$ characteristic $>\perp$ electrical- 
quantity $<$ physical $><$ categorical $><$ physics $>$ ". This is herein called as the word episodic symbolization.

With word episodic symbolization, all words and phrases of multiple meanings in a sentence or clause can be stored in analogy to the form of the noun "charge". In translation, all words and phrases of multiple meanings in the sentence or clause are directly transformed into the complex stored form from the databank. Then, according to the contents in the $<>$, the computer makes additional processing of the sentence or clause. If the sentence is about physics, then the electrical-quantity $<$ physical $><$ categorical $><$ physics $>$ matches the meaning of "charge". If the sentence is about the job, then the duty <zoological $><$ organizational $><$ behavioral $>$ matches the meaning of "charge". In this way, it is prospective for the word episodic symbolization to help discern one correct meaning for the word and phrase of multiple meanings in sentence.

Word episodic symbolization can store the various episodic associations of a word with the characteristic episodic symbols affiliated with the word, make the computer easy to process the various episodic meanings in the sentence, which would bring about significant progression to the automatic comprehension and translation of language.

\section{Discussions}

Based on the semantic/syntactic/episodic linguistic model, it has been derived the three corresponding principles for machine translation, respectively as first to establish the dictionary of words/phrases, second to translate the grammar, and third to determine the meaning of some words/phrases of multiple meanings by statistical associations with others [2]. In this article, it is further demonstrated three types of episodic associations for this neural model of language, prospectively to improve the statistical machine translation, concretely as the behavioral classification of the living/natural words, the categorization of sentence/paragraph and the collection of frequent word-pairs.

It is noted that the word episodic symbolization herein can store the various episodic associations of a word with the characteristic episodic symbols affiliated with the word, which would be a breakthrough for the computer to process the various episodic meanings in the sentence.

In this regard, due to the prospective improvement of statistical translation by these methods, the third principle of machine translation should correspondingly be improved by using these three methods, as the followings: third to determine the meaning of some words/phrases of multiple meanings by episodic associations with others using the behavioral classification of the living/natural words, the categorization of sentence/paragraph and the collection of frequent word-pairs.

It is necessary to point out that there are still some other methods able to improve the statistical translation, such as using the visual, auditory inputs and associations, and so on. This article only deals with the improvement of statistical 
translation prospectively brought about from the three new type of episodic associations demonstrated here, while does not consider those from others.

\section{Conclusion}

In this article, it is newly demonstrated three types of episodic associations for the semantic/syntactic/episodic neural model of language, prospectively to help and improve the statistical translation. It is adopted the behavioral characteristics both as zoological/organizational/physical/categorical and affective/behavioral/logic/characteristic/changing to further classify the living/natural nouns and verbs, the affective/behavioral/logic/characteristic/changing/spatial/temporal characteristics to the living/natural adjectives and adverbs, so as to help discern the meaning of words/phrases of multiple meanings with these episodic associations within the sentence or clause. For the same purpose, it is also classified the sentence and paragraph into the category of natural/social subjects such as physics, biology, art, economy, and so on, so as to help discern the meaning of words/phrases of multiple meanings with episodic associations. It is further required to collect the frequent word-pairs during statistical translation, so as to help determine the episodic associations of some prepositions or some terminal "which" clauses. Finally, it is suggested to use word episodic symbolization to apply them to computer. In these respects, it is herein improved the third principle of machine translation as third to determine the meaning of some words/ phrases of multiple meanings by episodic associations with others using the behavioral classification of the living/natural words, the categorization of sentence/paragraph and the collection of frequent word-pairs.

\section{Highlights}

- Behavioral classifying the living/natural words helps the statistical translation.

- Sentential/paragraphic categorization is useful to statistical translation.

- Collection of frequent word-pairs is necessary to statistical translation.

- Word episodic symbolization is useful to make databank in computer.

- These methods can help the statistical translation significantly.

\section{Conflict of Interest}

The author declares no conflict of interest or financial support for this work.

\section{Acknowledgments}

It is herein acknowledged that Mingxun Cai paid the Open Access publication fee of this paper.

\section{References}

[1] Cai, Z.J. (2015) Semantic Memory Association, Procedural Grammar Syntax and Episodic Modality Coordination as Three Interactive Neural Processes Organizing 
Language: A Model. Open Access Library Journal, 2, Article ID: e1718. http://dx.doi.org/10.4236/oalib.1101718

[2] Cai, Z.J. (2016) Principles Derived from Neurolinguistics of Brain for Design of Translation Machines. Open Access Library Journal, 3, e2704. http://dx.doi.org/10.4236/oalib.1102704

[3] Pinker, S. (1991) Rules of Language. Science, 253, 530-535. https://doi.org/10.1126/science.1857983

[4] Ullman, M.T., Corkin, S., Coppola, M., Hickok, G., Growdon, J.H., Koroshetz, W.J. and Pinker, S. (1997) A Neural Dissociation within Language: Evidence that the Mental Dictionary Is Part of Declarative Memory, and that Grammatical Rules Are Processed by the Procedural System. Journal of Cognitive Neuroscience, 9, 266-276. https://doi.org/10.1162/jocn.1997.9.2.266

[5] Ullman, M.T. (2004) Contributions of Memory Circuits to Language: The Declarative/Procedural Model. Cognition, 92, 231-270. https://doi.org/10.1016/j.cognition.2003.10.008

[6] Maguire, G.A., Yu, B.P., Franklin, D.L. and Riley, G.D. (2004) Alleviating Stuttering with Pharmacological Interventions. Expert Opinion on Pharmacotherapy, 5, 15651571. https://doi.org/10.1517/14656566.5.7.1565

[7] Stager, S.V., Calis, K., Grothe, D., Bloch, M., Berensen, N.M., Smith, P.J. and Braun, A. (2005) Treatment with Medications Affecting Dopaminergic and Serotonergic Mechanisms: Effects on Fluency and Anxiety in Persons Who Stutter. Journal of Fluency Disorders, 30, 319-335. https://doi.org/10.1016/j.jfludis.2005.09.004

[8] Lan, J., Song, M., Pan, C., Zhuang, G., Wang, Y., Ma, W., Chu, Q., Lai, Q., Xu, F., Li, Y., Liu, L. and Wang, W. (2009) Association between Dopaminergic Genes (SLC6A 3 and DRD2) and Stuttering among Han Chinese. Journal of Human Genetics, 54, 457-460. https://doi.org/10.1038/jhg.2009.60

[9] Small, S.L. and Llano, D.A. (2009) Biological Approaches to Aphasia Treatment. Current Neurology and Neuroscience Reports, 9, 443-450. https://doi.org/10.1007/s11910-009-0066-x

[10] Berthier, M.L., Pulvermüller, F., Dávila, G., Casares, N.G. and Gutiérrez, A. (2011) Drug Therapy of Post-Stroke Aphasia: A Review of Current Evidence. Neuropsychology Review, 21, 302-317. https://doi.org/10.1007/s11065-011-9177-7

[11] Cape, E.G., Manns, I.D., Alonso, A., Beaudet, A. and Jones, B.E. (2000) Neurotensin-Induced Bursting of Cholinergic Basal Forebrain Neurons Promotes Gamma and Theta Cortical Activity Together with Waking and Paradoxical Sleep. The Journal of Neuroscience, 20, 8452-8461.

[12] Mena-Segovia, J., Sims, H.M., Magill, P.J. and Bolam, J.P. (2008) Cholinergic Brainstem Neurons Modulate Cortical Gamma Activity during Slow Oscillations. The Journal of Physiology, 586, 2947-2960. https://doi.org/10.1113/jphysiol.2008.153874

[13] Wang, L., Zhu, Z. and Bastiaansen, M. (2012) Integration or Predictability? A Further Specification of the Functional Role of Gamma Oscillations in Language Comprehension. Frontiers in Psychology, 3, 187. https://doi.org/10.3389/fpsyg.2012.00187

[14] Vidal, J.R., Freyermuth, S., Jerbi, K., Hamamé, C.M., Ossandon, T., Bertrand, O., Minotti, L., Kahane, P., Berthoz, A. and Lachaux, J.P. (2012) Long-Distance Amplitude Correlations in the High $\gamma$ Band Reveal Segregation and Integration within the Reading Network. The Journal of Neuroscience, 32, 6421-6434. https://doi.org/10.1523/JNEUROSCI.4363-11.2012

[15] Weiss, S. and Müller, H.M. (2013) The Non-Stop Road from Concrete to Abstract: 
High Concreteness Causes the Activation of Long-Range Networks. Frontier in Human Neuroscience, 7, 526. https://doi.org/10.3389/fnhum.2013.00526

[16] Miniscalco, C., Hagberg, B., Kadesjö, B., Westerlund, M. and Gillberg, C. (2007) Narrative Skills, Cognitive Profiles and Neuropsychiatric Disorders in 7-8-Year-Old Children with Late Developing Language. International Journal of Language and Communication Disorders, 42, 665-681. https://doi.org/10.1080/13682820601084428

[17] Ash, S., Menaged, A., Olm, C., McMillan, C.T., Boller, A., Irwin, D.J., McCluskey, L., Elman, L. and Grossman, M. (2014) Narrative Discourse Deficits in Amyotrophic Lateral Sclerosis. Neurology, 83, 520-528. https://doi.org/10.1212/WNL.0000000000000670

[18] Youse, K.M. and Coelho, C.A. (2009) Treating Underlying Attention Deficits as a Means for Improving Conversational Discourse in Individuals with Closed Head Injury: A Preliminary Study. Neurorehabilitation, 24, 355-364.

[19] DiLollo, A., Neimeyer, R.A. and Manning, W.H. (2002) A Personal Construct Psychology View of Relapse: Indications for a Narrative Therapy Component to Stuttering Treatment. Journal of Fluency Disorders, 27, 19-40. https://doi.org/10.1016/S0094-730X(01)00109-7

[20] Leahy, M.M., O’Dwyer, M. and Ryan, F. (2012) Witnessing Stories: Definitional Ceremonies in Narrative Therapy with Adults Who Stutter. Journal of Fluency Disorders, 37, 234-241. https://doi.org/10.1016/j.jfludis.2012.03.001

[21] Zhang, M., Duan, X.Y. and Chen, W.L. (2014) Bayesian Constituent Context Model for Grammar Induction. IEEE/ACM Transactions on Audio, Speech, and Language Processing, 22, 531-541. https://doi.org/10.1109/TASLP.2013.2294584

[22] Xiong, D.Y., Zhang, M. and Li, H.Z. (2011) A Maximum-Entropy Segmentation Model for Statistical Machine Translation. IEEE Transactions on Audio, Speech, and Language Processing, 19, 2494-2505. https://doi.org/10.1109/TASL.2011.2144971

[23] Xiong, D.Y., Zhang, M. and Wang, X. (2015) Topic-Based Coherence Modeling for Statistical Machine Translation. IEEE/ACM Transactions on Audio, Speech, and Language Processing, 23, 483-493. https://doi.org/10.1109/TASLP.2015.2395254

Submit or recommend next manuscript to OALib Journal and we will provide best service for you:

- Publication frequency: Monthly

- 9 subject areas of science, technology and medicine

- Fair and rigorous peer-review system

- Fast publication process

- Article promotion in various social networking sites (LinkedIn, Facebook, Twitter, etc.)

- Maximum dissemination of your research work

Submit Your Paper Online: Click Here to Submit

Or Contact service@oalib.com 